\title{
Response of the human intestine to high volume infusion
}

\author{
C Steadman, P Kerlin
}

\begin{abstract}
The motor patterns and luminal capacity of the human intestine should affect symptoms and resorption during pathological, massive small intestinal flow. Little is known of human intestinal motility in this situation. This study aimed at mimicking secretory diarrhoea (experimentally) in healthy volunteers by intrajejunal infusion of a non-absorbable iso-osmotic solution at $20 \mathrm{ml} / \mathrm{min}$. During the infusion intraluminal jejunal pressures and small intestinal transit times were measured. The infusion initially caused jejunal contractile activity similar to that of the fed state but this was replaced by discrete clusters of contractions (DCCs) after 29.1 ((SEM) 8.2) minutes. DCCs each lasted 38 ((SEM) 0.8 seconds) and were associated with colicky abdominal discomfort. Later, after 1400-1800 $\mathrm{ml}$ had been infused, distal jejunal pressure waves fell to $10 \mathrm{~mm} \mathrm{Hg}$ or less. Frequent fasting DCCs predicted earlier onset and more frequent DCCs during the infusion. Thus, the rate and volume of flow during simulated secretory diarrhoea determine the pattern of the small bowel pressure profile; eventually, a volume load is reached in which the small bowel acts as a poorly segmenting conduit resulting in very fast transit rates. (Gut 1994; 35: 641-645)
\end{abstract}

The motor patterns and luminal capacity of the human intestine should affect symptoms and resorption when massive small intestinal flow occurs in secretory diarrhoea. Little is known of human intestinal motility in this situation. Studies of volume-flow relations have shown that initially, as intraluminal flow increases, so does jejunal volume. When fluids enter at rates faster than $7 \mathrm{ml} / \mathrm{min}$ the small intestine seems to control its volume by disposing of fluid distally, ${ }^{1}$ probably at the expense of absorption. ${ }^{2}$ One mechanism for rapid intestinal transit may be the onset of specialised patterns of propulsive small intestinal motor activity. In animal models distinct patterns of jejunal contractile activity, such as clustered contractions occurring in a minute rhythm, are stimulated by loading the small intestine with both nutrient and non-nutrient substances. ${ }^{34}$ An alternative mechanism for rapid transit could be unimpeded flow of contents when the small intestine becomes distended and minimally compliant at high intraluminal volumes.

The colon compensates for high volume small intestinal flow by acting as a reservoir that retains secretions for resorption. ${ }^{5}$ Boluses of fluid greater than $250-500 \mathrm{ml}$ instilled directly into the caecum cause diarrhoea but whether the capacity of the colon increases by receptive relaxation when the small intestine is distended is uncer- tain. Small intestinal motility could affect colonic absorptive and storage capacity in determining the onset of diarrhoea.

We aimed at mimicking secretory diarrhoea experimentally in healthy volunteers by intrajejunal infusion. Our hypothesis was that because the human jejunum can limit its volume, it would respond to infusion with specialised patterns of phasic and tonic contraction induced by the large volumes of fluid entering its lumen. The response to the infusion was determined by measuring variations in jejunal intraluminal pressures (jejunal motility), small intestinal transit time, and the volumes that the intestine accommodated during the infusion.

\section{Methods}

\section{SUBJECTS}

Eight healthy male volunteers aged 18-31 years gave informed consent to participate in a protocol approved by the ethics and radiation safety committees of the Princess Alexandra Hospital. All subjects were free from any gastrointestinal symptoms and had normal physical examinations. The unpleasant nature of the studies meant that large numbers of volunteers could not be investigated.

EXPERIMENTAL DESIGN AND PROCEDURES

To reproduce the clinical circumstances of secretory diarrhoea, six of eight subjects drank three litres of the infusate during the evening of the day before the study day then fasted (last meal about $600 \mathrm{pm}$ on the day before the study). Each of these subjects developed watery diarrhoea, which had settled before the infusion was started on the day of study between 900 and $1000 \mathrm{am}$. Their responses were compared with those in two subjects who had no preparation other than a 12 hour fast. These subjects were studied without preparation as internal controls to see if preparation before the study significantly changed fasting contractile patterns or the response to the infusion (see analysis).

A multilumen catheter (outer diameter $5 \mathrm{~mm}$ ) incorporating a $2 \mathrm{~mm}$ infusion lumen, six capillary infusion lumens for sidehole manometry, and an inflatable balloon and weight tip, was placed in the jejunum using a previously described technique. ${ }^{6}$ The catheter was advanced under fluoroscopic guidance until the infusion outlet was in the third part of the duodenum. Beginning $5 \mathrm{~cm}$ distal to the infusion site, roughly at the ligament of Trietz, were six side hole manometric recording sites spaced $10 \mathrm{~cm}$ apart. After intubation the volunteers rested in bed. 
Intraluminal pressure changes were recorded using a low compliance pneumohydraulic capillary infusion system ${ }^{7}$ with on line pressure transducers. The analogue signals from the transducers were simultaneously recorded; those from the five most distal sites were electronically integrated to produce a motility index (area under the curve $-\mathrm{mm} \mathrm{Hg} / \mathrm{min}^{8}$ ) that was displayed on the real time record for $500 \mathrm{~ms}$ every minute. Fasting contractile activity was recorded until two episodes of phase three activity of the fasting migrating motor complex (MMC) had occurred or 4.5 hours had passed. Pressure recordings were continuous throughout.

To measure small intestinal transit the infusion into the third part of the duodenum was begun with $7 \mathrm{~g}$ of lactulose (Duphar Company, Holland) diluted in $30 \mathrm{ml}$ of water followed immediately by the infusate solution. Although the lactulose would have mixed with the infusate it was assumed that at least some of the lactulose bolus remained at the leading edge of the infused fluid as it moved in the aborad direction. Any influx of fluid into the intestine caused by the osmotic effects of the lactulose bolus was assumed to have added to the volume load. ${ }^{9}$ Before the lactulose was given two basal end expiratory breath hydrogen $\left(\mathrm{H}_{2}\right)$ samples were collected. Subsequent breath $\mathrm{H}_{2}$ samples were taken every five minutes until the concentration increased more than $20 \mathrm{ppm}$, indicating arrival of the lactulose in the colon. ${ }^{10}$ Breath $\mathrm{H}_{2}$ concentration was measured by gas chromatography using previously described methods. ${ }^{11}$

The infusion was started during phase two of the MMC. An infusion system produced a jejunal flow rate of $20 \mathrm{ml} / \mathrm{min}$; this high flow rate was chosen to mimic the flow of secretions in the Zollinger-Ellison syndrome ${ }^{12-14}$ in which secretory diarrhoea is caused by excessive secretion of the hormone gastrin. The infusion fluid was composed of polyethylene glycol 3350 (PEG) and electrolyte powder (Colonic Lavage Powder, Queensland Ethicals, Salisbury, Australia) dissolved in water in a concentration of $69.6 \mathrm{~g} / \mathrm{l}$ (PEG $60 \mathrm{~g} / \mathrm{l}$; sodium chloride $1.46 \mathrm{~g} / \mathrm{l}$; sodium bicarbonate $1.68 \mathrm{~g} / \mathrm{l}$; sodium sulphate (anhydrous) $5.68 \mathrm{~g} / \mathrm{l}$ ). This iso-osmotic, colonic lavage solution was chosen for known safety and because it is not absorbed. ${ }^{15}$ The infusion rate was controlled using a drip chamber and graduated bags of infusate filled with standard measuring cylinders.

The infusion was continued until each volun-



Figure 1: Manometric recording showing the rapid onset of irregular contractile activity that was the initial response to high volume jejunal infusion; later it was replaced, particularly in the distal jejunum, by repetitive discrete clustered contractions (DCCs). teer became symptomatic from the infusion with significant abdominal distension, colicky abdominal pain, nausea or the irresistible urge to defecate.

\section{ANALYSIS}

Pressure variations were taken to represent intestinal contractile activity. Recordings were visually inspected for patterns of motility. The criteria for the phases of the fasting small intestinal MMC were minimal or no contractile activity for phase 1 , irregular activity for phase 2 , and an uninterrupted series of contractions of $>3$ minutes duration followed by quiescence for phase 3 (an activity front). ${ }^{16}$ Discrete clusters of contractions (DCCs) were defined as groups of three or more contractions $>5 \mathrm{~mm} \mathrm{Hg}$ in amplitude, lasting less than 120 seconds, preceded and followed by $>20$ seconds of inactivity. Propagation of DCCs was judged to be present if they appeared sequentially over three or more recording sites, or over two recording sites if the pattern of contractions was the same.

Where possible, symptomatology during the infusion was correlated with patterns of contractile activity and small intestinal transit. Motility indices for each minute were averaged over all recording sites during each definable pattern of contractile activity. Motility indices judged to be significantly affected by artifact were excluded from the analysis.

The results of the studies in the two patients who did not receive preparation were included in the analysis as in both, the pattern of response to the infusion was similar to those who had previous preparation. Statistical comparisons were made using Student's $t$ test for paired data and correlations tested using univariate linear regression.

\section{Results}

\section{CONTRACTILE ACTIVITY}

All volunteers completed the protocol. The mean (SEM) infusion rate was $20.2(0.3 \mathrm{ml} / \mathrm{min})$ for 69-100 minutes (mean 80.8). Each of the six volunteers who had bowel preparation before study had two activity fronts (phase 3 of MMC) before the infusion. The other two had only one activity front during 260 and 278 minutes of fasting study. All phase 3 activity fronts had frequencies $>10 / \mathrm{min}$ showing that the catheter position was stable in the jejunum.

In six subjects the start of the infusion caused increased irregular contractile activity that was similar to a fed pattern. In three subjects, phase 3 like bursts of contractions occurred almost immediately after the infusion was commenced and preceded the irregular activity.

In the six who displayed the irregular pattern, repetitive DCC activity followed. DCCs began mean (SEM) 29.1 (8.2) min after the start of the infusion (Fig 1). They were most commonly seen in the recordings from distal jejunal sites but were also recorded in the proximal jejunum, which tended to retain the irregular fed like pattern. DCCs did not occur exclusively as periods of irregular activity sometimes recurred. 


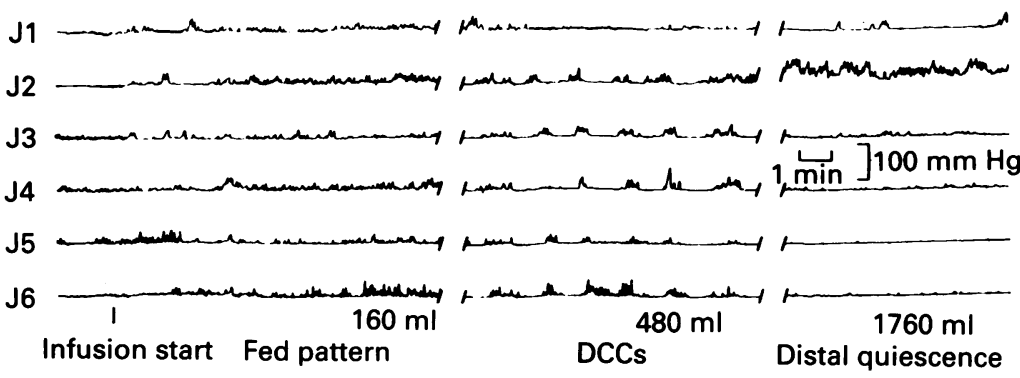

Figure 2: Manometric recording showing the three phases of contractile activity during jejunal infusion: (1) irregular, (2) discrete clustered contractions (DCCs), and (3) late, distal jejunal quiescence. approximately 1400 to $1800 \mathrm{ml}$ of fluid had been infused.

\section{CONTRACTILE ACTIVITY AND TRANSIT}

All subjects had a rise in breath $\mathrm{H}_{2}$. This occurred during either irregular contractile activity or DCCs. The mean (SEM) small intestinal transit time was 35 (4) minutes - that is, average of $700 \mathrm{ml}$ infused. Small intestinal transit times did not correlate with the onset of DCC or any other specialised pattern of contractions that might have been propelling contents to the colon. Small intestinal transit times were similar among the group. Colonic transit times (estimated by subtracting small intestinal transit time from the time of onset of the irresistible urge to defecate) were also closely grouped (Fig 4). DCC activity almost immediately after the infusion was commenced and DCCs persisted throughout the infusion.

Individual DCCs had a mean (SEM) duration of $38(0 \cdot 8)$ seconds, appeared simultaneously over multiple sites or were rapidly propagated at $60-120 \mathrm{~cm} / \mathrm{min}$ (but as rapidly as $300 \mathrm{~cm} / \mathrm{min}$ ) over distances up to $40 \mathrm{~cm}$ (Fig 2). Sometimes DCCs seemed to occur distally before proximally but no clear retrograde propagation was seen. DCCs were more frequent during the infusion than in phase 2 of the fasting MMC (mean (SEM) $\mathrm{DCC} /$ minute during infusion $0.57(0.11) v 0.24$ $(0.06)$ during phase $2 ; \mathrm{p}<0.001)$. The rate of fasting DCCs correlated with the rate of intrainfusional DCCs $\left(r^{2}=0.89 ; \mathrm{p}<0.001\right.$, Fig 3). Those with many fasting DCCs also tended to show repetitive DCCs sooner after the infusion was started $\left(r^{2}=0.48 ; \mathrm{p}=0.055\right)$. Analysis of mean motility indices showed that contractile activity was greatest during phase 3 of the fasting MMC. The mean motility index during the irregular contractile activity early in the infusion was greater than that during fasting phase 2 activity $(\mathrm{p}<0.05)$.

In three subjects, including the two who had not been prepared before the study, the infusion was stopped because of symptoms during DCC activity. These subjects had the shortest periods of study (69-76 minutes).

The third feature of the sequential jejunal response occurred in the five other subjects who showed a variable decrease in the number and amplitude of contractions until, in the three or four most distal recording sites, pressure waves fell to approximately $10 \mathrm{~mm} \mathrm{Hg}$ or less (Fig 2). Declining pressure fluctuations occurred after

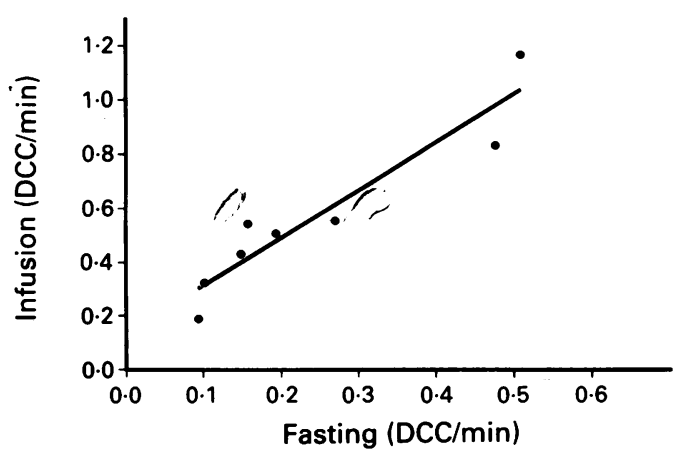

Figure 3: The rate of fasting discrete clustered contractions directly correlated with the higher rate seen during jejunal infusion $\left(\mathrm{r}^{2}=0.89 ; p<0 \cdot 001\right)$. Thus, frequent fasting DCCs point to a low threshold for stimulated DCCs.

\section{SYMPTOMS}

The infusion caused symptomatic, readily apparent abdominal distension in all subjects. The onset of DCCs was associated with the onset of colicky abdominal discomfort but individual 'DCCs did not occur at exactly the same time as bouts of colic. One subject had a brief episode of colicky pain and DCCs then became asymptomatic. He had a longer small intestinal transit time (60 minutes) and time to defecation (130 minutes) than other subjects and was the most distended at the end of the study $(2000 \mathrm{ml}$ infused).

Two subjects became pale and nauseated with symptoms suggesting upper intestinal stasis but they had small intestinal transit times of 25 and 35 minutes and both retained the urge to defecate. Vomiting terminated the study in one of them and obscured intestinal contractile events until a phase 3 activity front traversed all recording sites.

\section{Discussion}

There is limited information on the motility of the human small intestine in secretory diarrhoea. Our results support the hypothesis that the intestinal response to high volume infusion is a reproducible sequence. The main purpose of the small intestine is salvage of luminal secretions but as the capacity and absorptive reserve of the intestine are exceeded, mechanisms for the dis-

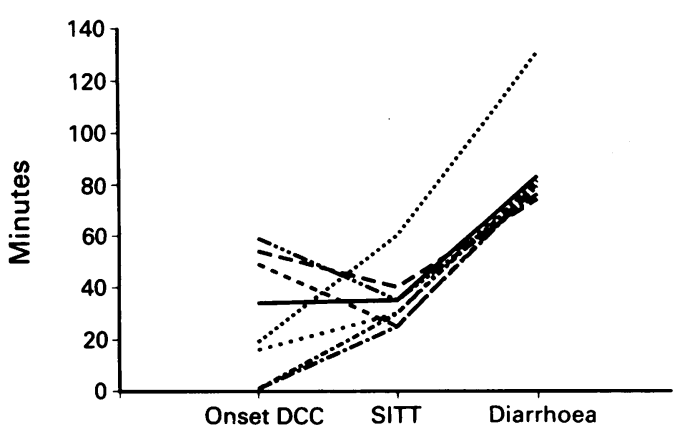

Figure 4: Intestinal transit times (SITT - small intestinal transit time; colonic transit time $($ approximate $)=$ time to defecation) varied little among subjects suggesting that the volume entering the intestinal lumen was a more important determinant of flow than individual contractile patterns. The onset of DCCs did not correlate with SITT. 
posal of fluid as diarrhoea come into play. ${ }^{217} \mathrm{We}$ saw early intrainfusional contractile patterns that were similar to postprandial activity (fed state pattern) and they interrupted the fasting MMC. The phase 3 like bursts that preceded irregular activity in these subjects could have occurred coincidentally or been part of the induced response.

Merging with the fed state pattern were DCCs that were the second event in the sequence we saw. There was a direct correlation between the rate of fasting jejunal DCCs and the higher rate during infusion and the two subjects with frequent fasting DCCs had the most rapid onset of repetitive DCCs. Thus, the frequency of fasting DCCs seemed to be a marker for greater jejunal sensitivity to distension. DCCs, initially seen infrequently in the human jejunum during late phase 2 of the interdigestive $M M C,{ }^{18}{ }^{19}$ were seen frequently in humans in response to eating when partial small bowel obstruction was causing intestinal distension..$^{20}$ In animal models of diarrhoea propagated DCCs have been induced by loading the jejunum with fluid and overfeeding with grain. ${ }^{321}$ When DCCs occurred regularly at 1-2 minute intervals they were called 'minute rhythm's and were thought to be distally propulsive as they are in the normal ileum. ${ }^{22-24}$ In contrast, DCCs in the jejunum seem to be a nonspecific response to distension caused by obstruction or intraluminal loading and they are probably not propulsive in many cases.

That DCCs are non-specific, and possibly not pathological, is supported by previous findings in chronic diarrhoeal illness ${ }^{25}$ and other circumstances. ${ }^{26-29}$ Luminal flow (rather than DCCs) was the main determinant of small intestinal transit in this study as transit times were similar among subjects regardless of contractile patterns. The significance of propagated and non-propagated DCCs is, however, probably different with respect to transit.

Despite these findings a lower threshold for DCCs may affect the response to secretion and point to a greater likelihood of symptomatic sensitivity to intestinal distension as occurred in this study. In irritable bowel syndrome frequent jejunal DCCs occur with similar colicky abdominal pain. ${ }^{3031}$

Reduced distal jejunal pressure fluctuation followed DCCs after infusion of $1400 \mathrm{ml}$ (or more), presumably because orad intestinal distension decreased aborad muscle tone resulting in a more capacious intestinal lumen and a Bainbridge reflex response characterised by reduced phasic contractile activity distally. ${ }^{32}$ Reduced pressure fluctuations, however, could also have resulted from the small intestine being stretched to the point of mechanical disadvantage impairing contraction and causing the lumen to behave as an open system. There was no evidence of obstruction as diarrhoea occurred promptly in all subjects. Either way, it seemed that eventually the small bowel became a poorly segmenting conduit facilitating rapid distal flow under the impetus of proximal jejunal peristalsis (mimicking a situation when secretion exceeds $7 \mathrm{ml} /$ min). ${ }^{1}$ In overfed animals, jejunal motor quiescence was typical of periods when diarrhoea was most severe. ${ }^{23}$
Human jejunal motor quiescence has also been seen under other circumstances. Infusion of glycochenodeoxycholic acid at lower rates (about $5 \mathrm{ml} / \mathrm{min})^{33}$ caused motor quiescence. Whether relaxation is regulated or occurs because of intestinal muscle fatigue is unclear as in animals purged with castor oil or magnesium sulphate, the small intestine became floppy and subsequent in vitro experiments showed that small intestinal muscle strips were rapidly fatigued by repetitive stimulation. ${ }^{34}$ It does not seem probable that relaxation could have permitted the distal small intestine to become a reservoir because in previous studies at steady infusion rates, jejunal volumes were approximately $1 \cdot 6$ times greater than ileal volumes for any given rate. ${ }^{1}$

This study is relevent to the pathophysiology of secretory diarrhoea where secretions cause high intestinal flow. Those who are prone to the early onset of diarrhoea may have maladaptive intestinal motility responses such that fed state contractile activity is replaced by DCCs or motor quiescence at comparatively low intraluminal volumes.

We would like to thank Mrs Donna McIntyre RN for technical assistance and Mrs Valerie Shaw for typing the manuscript.

1 Dillard RL, Eastman H, Fordtran JS. Volume-flow relationship during the transport of fluid through the human small intestine. Gastroenterology 1965; 49: 58-66.

2 Love AH, Mitchell TG, Phillips RA. Water and sodium absorption in the human intestine. F Physiol 1968; 195: 133-40.

3 Fleckenstein P, Bueno L, Fioramonti J, Ruckebusch Y. Minute rhythm of electrical spike bursts of the small intestine in different species. Am $f$ Physiol 1982; 242: intestine

4 Ruckebusch Y, Bueno L. The effect of feeding on the motility of the stomach and small intestine in the pig. Brf Nutr 1976; 35: $397-405$.

5 Debongnie JC, Phillips SF. Capacity of the human colon to absorb fluid. Gastroenterology 1978; 74: 698-703.

6 Kerlin P, Tucker R, Phillips S. Rapid intubation of the ileocecal region in man. Aust NZ F Med 1983; 13: 591-3.

7 Arndorfer RC, Stef JJ, Dodds WJ, Linehan JH, Hogan WJ. Improved infusion system for intraluminal esophageal manometry. Gastroenterology 1977; 73: 23-7.

8 Kerlin P, Zinsmeister A, Phillips S. Motor responses to food of the ileum, proximal colon and distal colon of healthy humans. Gastroenterology 1983; 84: 762-70.

9 Holgate AM, Read NW. Relationship between small bowel transit time and absorption of a solid meal. Influence of transit time and absorption of a solid meal. Influence of Sci 1983; 28: 812-9.

10 Bond JH, Levitt MD. Investigation of small bowel transit time in man utilizing pulmonary hydrogen $\left(\mathrm{H}_{2}\right)$ measurements. f Clin Lab Med 1975; 85: 546-55.

11 Kerlin P, Wong L. Breath hydrogen testing in bacterial overgrowth of the small intestine. Gastroenterology 1988; 95: 982-8.

12 Rambaud JC, Modigliani R, Emonts P, Patuchansky C, Vidon $\mathrm{N}$, Besterman $\mathrm{H}$, et al. Fluid secretion in the duodenum and intestinal handling of water and electrolytes in ZollingerEllison syndrome. Dig Dis Sci 1978; 23: 1089-97.

13 Malagelada JR. Pathophysiological responses to meals in the Zollinger-Ellison syndrome: 1. Paradoxical postprandial inhibition of gastric secretion. Gut 1978; 19: 284-9.

14 Malagelada JR. Pathophysiological responses to meals in the Zollinger-Ellison syndrome: 2 . Gastric emptying and its Zollinger-Ellison syndrome: 2. Gastric emptying

15 Davis GR, Santa Ana CA, Morawski SG, Fordtran JS Inhibition of water and electrolyte absorption by poly ethylene glycol (PEG). Gastroenterology 1980; 79: 35-9.

16 Kerlin P, Phillips S. Variability of motility of the ileum and jejunum in healthy humans. Gastroenterology 1982; 82: 694-700.

17 Shehadeh Z, Grantham RN, Brecher GA, Jacobson ED. Effects of infusion rate and osmolarity on volumogenic diarrhea. Gastroenterology 1969; 57: 24-9.

18 Fleckenstein $P$. Migrating electrical spike activity in the fasting human small intestine. Dig Dis 1978; 23: 769-75.

19 Fleckenstein P, Oigaard A. Electrical spike activity in the human small intestine. A multiple electrode study of fasting diurnal variations. Dig Dis 1978; 23: 776-80.

20 Summers RW, Anuras S, Green J. Jejunal manometry patterns in health, partial intestinal obstruction and pseudopatterns in health, partial intestinal obstruction and

21 Ruckebusch Y, Bueno L. Electrical activity of the ovine jejunum and changes due to disturbances. Dig Dis 1975; 20: jo27-34. 
22 Phillips SF. Motility of the ileocolonic junction. Gut 1988; 29: 390-406

23 Ehrlein HJ, Schemann M, Siegle ML. Motor patterns of small intestine determined by closely spaced extraluminal transducers and videofluoroscopy. Am f Physiol 1987; 253: G259-67.

24 Alvarez WC, Mahoney LJ. Peristaltic rush in the rabbit. Am f Physiol 1924; 69: 211-25.

25 Kellow J, Phillips S, Miller L, Osterholm M, MacDonald K. Abnormalities of motility and absorption in an outbreak of Abnormalities of motility and absorption in an out
chronic diarrhea. Gastroenterology 1985; 88: 1442 .

26 Berseth CL. Small intestinal motility in neonates: response to feeding. Gastroenterology 1989; 96: A42.

27 Berseth CL, Nordyke C. Gestational maturation of small intestinal motility during fasting in preterm and term infants. Gastroenterology 1988; 95: 857.

28 Coremans G, Chaussade S, Janssens J, Vantrappen G, Ceccatelli P. Stimulation of propulsive motility patterns by cisapride in the upper gut of man. Dig Dis Sci 1985; 30: 765 .
29 Camilleri M. Jejunal manometry in distal subacute mechanical obstruction: significance of prolonged simultaneous contractions. Gut 1989; 30: 468-75.

30 Horowitz L, Farrar JT. Intraluminal small intestinal pressures in normal patients and in patients with functional gastro-
intestinal disorders. Gastroenterology 1962; 42: 455-64.

31 Kellow JE, Phillips SF. Altered small bowel motility in irritable bowel syndrome is correlated with symptoms. irritable bowel syndrome is corr

32 Rouillon JM, Azpiroz F, Malagelada J-R. Responsiveness of the jejunum to intestino-intestinal reflexes in humans. the jejunum to intestino-intestin

33 Penagini R, Spiller RC, Misiewicz JJ, Frost PG. Effect of ileal infusion of glycochenodeoxycholic acid on segmental transit, motility and flow in the human jejunum and ileum. Gut 1989; 30: 609-17.

34 Alvarez WC, Taylor FB. Changes in rhythmicity, irritability and tone in the purged intestine. F Pharmacol Exp Ther 1917; 10: $365-77$. 